\title{
SOME THEOREMS CONNECTED WITH ABEL'S THEOREM ON THE CONTINUITY OF POWER SERIES
}

\author{
By G. H. HARDY.
}

[Received March 31st, 1906.-Read April 26th, 1906.-Roceived in revised form May 6th, 1906.]

1. It will probably make the object of this paper more easily intelligible if, at the risk of repeating a certain number of well known facts, I preface it with a brief historical résumé.

In his famous memoir on the Binomial Series Abel proved that, if a series $\Sigma a_{n}$ is convergent, the series $\Sigma a_{n} x^{n}$ is convergent for all positive values of $x$ less than unity, and represents a function $f(x)$ which is continuous for all such values of $x$, unity included.*

An alternative proof of Abel's theorem was given later by Dirichlet. $t$

Stated in the language of the modern theory of functions, Abel's theorem runs: "If a power series in $x$ converges to the sum $s$ at a point $P$ on its circle of convergence, and $f(x)$ is the function represented by the series within the circle, then $f(x)$ tends to the limit $s$ when $x$ tends to $P$ along a radius vector from the origin."

This theorem has proved the starting point for a considerable number of later researches. Stolz was the first to prove that the result still holds if $x$ tends to $P$ along any path which lies entirely within the circle of convergence. $\ddagger$ At a later date Pringsheim returned to the subject in a very instructive memoir, $\$$ in which he shows that Abel's proof suffices to prove not only the continuity of $f(x)$, but also the uniform convergence of the series $\Sigma a_{n} x^{n}$ throughout the interval $(0,1)$. Of this the continuity of $f(x)$ for $x=1$ is a corollary ; but Abel had really proved more than mere continuity, and Pringsheim justly remarks that Dirichlet's proof is inferior to Abel's in that it obscures this fundamental point.

This is not the only direction in which Abel's theorem has been generalised. The property of the special function $x^{n}$, upon which Abel's

- Crelle, Bd. I. ; Gíuvres, T. r., p. 223.

† Liouville, Sér. 2, T. vir. ; Werke, Bd. II., p. 305.

$\ddagger$ Zeitschr. f. Math., Bd. xx., p. 370, and Bd. Xxxx., p. 127. This statenuent is sumewhat loose; see $\$ 4$.

§ Lü̈uchener. Sitzlngsberichte, 1897 , p. 343. 
proof was based, was simply that expressed by the inequality

$$
x^{n} \geqslant x^{n+1} \quad(0 \leqslant x \leqslant 1),
$$

and it was at once suggested that similar theorems must hold for more general classes of series of the type $\sum a_{n} f_{n}(x)$. And, in fact, Dirichlet and Dedekind* arrived at the following results, which for the sake of brevity I state on the hypothesis that the functions $f_{n}(x)$ are real functions of $x$ defined for the interval $0 \leqslant x \leqslant 1$.

(a) If

$$
f_{n}(x) \geqslant f_{n+1}(x) \geqslant 0 \quad(0 \leqslant x \leqslant 1),
$$

and $\Sigma a_{n}$ is convergent, then $\Sigma a_{n} f_{n}(x)$ is convergent and, if every $f_{n}$ is continuous, the sum of the series is a continuous function of $x$.

(b) If $\Sigma a_{n}$ oscillates between finite limits of indetermination,

$$
f_{n}(x) \geqslant f_{n+1}(x), \text { and } \lim f_{n}=0,
$$

then $\sum a_{n} f_{n}(x)$ is convergent; and, if every $f_{n}$ is continuous, the sum of the series is a continuous function of $x$.

Dirichlet and Dedekind were concerned mainly with applications of these theorems to Dirichlet's series, and pass somewhat lightly over the general properties of series which are involved in them. Their exposition is also obscured to some extent by the fact that they do not utilize the notion of uniform convergence. I have therefore discussed the question further in $\$ 2$, and have stated a few theorems which summarize the conclusions which can be drawn from the discussion. I cannot claim any particular originality for these theorems, but, so far as I know, they have not, in the form in which I state them, been included in any published work. They would naturally suggest themselves to any one who undertook a careful analysis of the various theorems stated in this section, and Prof. Bromwich informs me that he has himself included Theorem I. $a$ in a tract on the theory of series which will ultimately form one of the Cambridge Tracts in Mathematics and Mathematical Physics.

I have also included in $\$ \$ 3,4$ some applications of these theorems which do not appear to have been noticed hitherto, and in $\$ 5$ I have discussed a passage in Kronecker's Vorlesungen iiber Integrale which is concerned with the subject, but appears to contain serious errors.

There is yet another form of generalisation of Abel's theorem which has occupied the attention of mathematicians. It may happen that the series $\sum a_{n} x^{n}$ is divergent at a point on the circle of convergence, but is capable of "summation" by one or other of the methods furnished by 
the theory of divergent series, Cesàro's method of mean values, or Borel's method of exponential summation, or one of the various generalisations of either method. And it results from the combined researches of a number of writers that, if $\Sigma a_{n}$ has the sum $s$ when summed according to any of these methods, then $f(x)$ tends to the limit $s$ when $x$ tends to the point in question on the circle of convergence by any path subject to certain restrictions. In the latter part of the paper I have occupied myself with series summable by Cesàro's method. The theorem for such series which corresponds to Abel's original theorem was first proved by Frobenius, ${ }^{*}$ and states that, if

and

$$
s_{n}=a_{0}+a_{1}+\ldots+a_{n}
$$$$
\lim _{n=\infty} \frac{s_{0}+s_{1}+\ldots+s_{n}}{n+1}=s
$$

then

$$
\lim _{x=1} f(x)=s \text {. }
$$

I have attempted to prove a general theorem which shall stand to this theorem in the same relation as Theorem I. to Abel's theorem. This theorem (Theorem II.) is the principal result of the paper: it will be found in $\$ 6$.

Finally, I have illustrated some of the most obvious applications of this general theorern, and I have indicated some further questions which are naturally suggested, but which I cannot profess to have completely solved.

I may remark that I was led to this investigation by considering various problems concerning the limits approached by the $q$-series of elliptic functions, when $q$ tends to a point on the unit circle, and a number of my illustrations are furnished by $q$-series. But $I$ have not in this paper attempted to treat any such particular class of problems systematically.

2. Theorem I. a.-If $f_{0}(x), f_{1}(x), f_{2}(x), \ldots$ is a series of real finite positive functions $\dagger$ such that

$$
f_{n}(x) \geqslant f_{n+1}(x) \quad(0 \leqslant x \leqslant 1),
$$

- Crelle, Bd. Lxxxix., p. 262.

+ A fuite function (fonction borree) is a function whose absolute value is, throughout the interval of variation of the independent variable, less than a constant $K$. It would obviously be enough to assert that $\left|f_{0}\right|<h^{-}$. 
and $\Sigma a_{n}$ is any convergent series, then the series $\Sigma a_{n} f_{n}(x)$ is uniformly convergent throughout the interval $(0,1)$.

For

(1) $\sum_{\nu=m}^{n} a_{\nu} f_{\nu}=\sum_{\nu=m}^{n-1}\left(a_{m}+a_{m+1}+\ldots+a_{\nu}\right)\left(f_{\nu}-f_{v+1}\right)+\left(a_{m}+a_{m+1}+\ldots+a_{n}\right) f_{n}$.

Choose $m_{0}$ so that, for $\nu \geqslant m \geqslant m_{0}$,

$$
\left|a_{m}+a_{m+1}+\ldots+a_{\nu}\right|<\epsilon \text {. }
$$

Then

$$
\left|\sum_{\nu=m}^{n} a_{\nu} f_{\nu}\right|<\epsilon f_{m}<\epsilon M,
$$

where $M$ is the maximum of $f_{0}(x)$ in the range $(0,1)$. The theorem is therefore proved.

Corollary.-If the functions $f_{n}(x)$ are continuous, the series $\Sigma a_{n} f_{n}(x)$ represents a function of $x$ continuous throughout the interval $0 \leqslant x \leqslant 1$.

Theorex I. a 1.-If the restriction that $f_{n}$ is real and positive is removed, and the condition (1) is replaced by the condition that

$$
\sum_{m}^{n}\left|f_{\nu}(x)-f_{v+1}(x)\right|<K,
$$

where $K$ is a constant, then the series $\Sigma a_{n} f_{n}$ is still uniformly convergent.*

We first observe that the existence of such a constant $K$ involves that of a constant $L$, such that $\left|f_{n}(x)\right|<L$, for all values of $x$ and $n$. For

$$
\left|f_{n}(x)\right| \leqslant\left|f_{0}(x)\right|+\sum_{0}^{n-1}\left|f_{v}(x)-f_{v+1}(x)\right|<M+K .
$$

Hence $\quad\left|\sum_{m}^{n} a_{\nu} f_{\nu}\right|<\epsilon\left\{\sum_{n}^{n-1}\left|f_{\nu}-f_{\nu+1}\right|+\left|f_{n}\right|\right\}<\epsilon(M+2 K)$,

and the result follows as before.

CoroLLARY.-If the functions $f_{n}$ are continuous, the sum of the series is continuous.

An obvious generalisation is-

Theorem I. a 2. - The conclusions of the preceding theorems and corollaries still hold if the terms of the series $\Sigma a_{n}$ are functions of $x$, provided the series is uniformly convergent, and (in the corollaries) the functions $a_{n}$ are continuous.

- We may suppose either that $f_{n}$ is a complex function of a real variable, or a function of a complex variable; in the latter case the interval $(0,1)$ must be replaced by a region. 
These theorems all arise from the Theorem (a) of Dirichlet-Dedekind. It is with this rather than with Theorem (b) that I am concerned in this paper; but the latter also raises interesting questions.

Theorem I. $b .-$ If the functions $f_{n}(x)$ satisfy, in addition to the conditions of $I$., the condition $\lim _{n=\infty} f_{n}(x)=0$, and if $\Sigma a_{n}$ oscillates between finite limits of indetermination, ${ }^{*}$ then the series $\Sigma a_{n} f_{n}$ is uniformly convergent.

In the first place there is a number $K$ such that

$$
\left|a_{m}+a_{m+1}+\ldots+a_{\nu}\right|<K
$$

for all values of $m$ and $\nu$. In the second place $f_{n}(x)$ is a function of $x$ which never increases as $n$ increases, and whose limit zero is a continuous function of $x$. The convergence of $f_{n}(x)$ to its limit is therefore uniform, $\dagger$ and we can choose $m_{0}$ so that, for $m \geqslant m_{0}$, and for all values of $x$,

$$
\left|f_{n}(x)\right|<\epsilon \text {. }
$$

The theorem now follows immediately from (1).

Corollary.-If the functions $f_{n}$ are continuous, the sum of the series $\Sigma a_{n} f_{n}(x)$ is a continuous function of $x$.

TheоRem I. $b$ 1.-If the restriction that the functions $f_{n}(x)$ are real and positive is removed, and the conditions to which they are subject are replaced by the condition that the series $\Sigma\left|f_{n}(x)-f_{n+1}(x)\right|$ is convergent, the series $\Sigma a_{n} f_{n}$ is convergent.

Theовем I. $b$ 2.-If in addition the functions $f_{n}$ are continuous and either of the equivalent conditions (i.) that the series $\Sigma\left|f_{n}-f_{n+1}\right|$ is uniformly convergent, or (ii.) that its sum represents a continuous function of $x$, is satisfied, the series $\Sigma a_{n} f_{n}$ will be uniformly convergent and continuous.

TheоReM I. $b$ 3.-The preceding conclusions are not affected if the $a_{n}$ 's are functions of $x$, provided a constant $K$ exists such that

$$
\left|a_{0}+a_{1}+\ldots+a_{n}\right|<K
$$

for all values of $n$ and $x$, and (if the continuity of the series is asserted) the functions $a_{n}$ are continuous.

These theorems follow at once by trifling modifications of the preceding arguments. It will be seen that the series of theorems I. $b, b 1, b 2, b 3$ runs almost, though not exactly, parallel to the series I. $a, a 1, a 2$.

* I.c., $\left|a_{0}+a_{1}+\ldots+a_{n}\right|<K$.

+ Dini, Grundlagen, pp. 148, 149. The corollary is substantially Dedekind's theorem: his proof is less simple, owing to the fact that he does not employ the notion of uniform convergenoe. 
3. Of the preceding theorems those of which the applications are most interesting are I. $a$ and its extension I. $a 1$.

Since $f_{n} \geqslant f_{n+1}, f_{n}$ tends to a limit for $n=\infty$ for all values of $x$; but in general it will not tend uniformly to this limit, and the limit will not be a continuous function of $x$. In the most important applications such a non-uniformity or discontinuity occurs at one or other end of the interval $(0,1)$, and the interest of the theorem lies in its application to establish the continuity of the series $\Sigma a_{n} f_{n}$ at this end. Thus

(i.) If

$$
f_{n}(x)=x^{n}, \quad f_{n} \geqslant f_{n+1},
$$

$$
\lim f_{n}=0(0 \leqslant x<1), \quad \lim f_{n}=1(x=1),
$$

and we obtain Pringsheim's form of Abel's theorem.

(ii.) If

$$
\begin{gathered}
f_{n}(x)=n^{-x}, \quad f_{n} \geqslant f_{n+1}, \\
\lim f_{n} \Rightarrow 0(0<x \leqslant 1), \quad \lim f_{n}=1 \quad(x=0),
\end{gathered}
$$

and we deduce that the Dirichlet's series

$$
\frac{a_{1}}{1^{x}}+\frac{a_{2}}{2^{x}}+\frac{a_{3}}{3^{x}}+\ldots
$$

is uniformly convergent throughout $(0,1)$, and so continuous for $x=0$, which is one of the Dirichlet-Dedekind theorems.

(iii.) If (denoting the independent variable now by q) we take

$$
\begin{aligned}
& f_{i n}(q)=\frac{q^{n}}{1+q^{n}}, \\
& \text { so that } \\
& f_{n}-f_{n+1}=\frac{q^{n}(1-q)}{\left(1+q^{n}\right)\left(1+q^{n+1}\right)} \geqslant 0, \\
& \text { and } \\
& \lim _{n=\infty} f_{n}=0(q<1),=\frac{1}{2}(q=1),
\end{aligned}
$$

and we deduce that, if $\Sigma a_{n}$ is convergent,

$$
\lim _{q=1} \Sigma \frac{a_{n} q^{n}}{1+q^{n}}=\frac{1}{2} \Sigma a_{n}
$$

numerous applications of this result [and the similar results for $\Sigma a_{n} q^{\prime \prime} /\left(1+q^{2 n}\right), \ldots$ ] may be mudo in the theory of elliptic functions. For instance, from

we deduce

$$
\log k=\log 4 \sqrt{ } q+4 \Sigma \frac{(-q)^{n}}{n\left(1+q^{n}\right)} *
$$

as may be verified independently.

(iv.) Let us next consider the series

$$
\Sigma^{n n n_{n} q_{-}^{n}(1-q)}{ }_{1-q^{n}}=\Sigma_{1+q+q^{2}+\ldots+q^{n-1}}^{n a_{n} q^{n}}
$$

Here

$$
\begin{gathered}
f_{n}(q)=\frac{n q^{n}}{1+q+q^{2}+\ldots+q^{n-1}}, \\
f_{n}(q)-f_{n+1}(q)=\underset{\left(1-q^{n}\right)\left(1-q^{n+1}\right)}{(1-q)^{2} q^{n}}\left(n-1-q-\ldots-q^{n-1}\right) \geqslant 0 .
\end{gathered}
$$

- Jacobi, Fundamenta Nova, p. 103. 
We deduce that

$$
\lim _{q=1}(1-q) \Sigma \frac{n a_{n} q^{n}}{1-q^{n}}=\Sigma a_{n}
$$

provided only the latter serics is convergent. This result has been proved (by a special method depending upon integrals) by Franel.* Similar results may, of course, be proved for such series

as

For instance, from

$$
\Sigma \frac{2 n a_{n} q^{n}}{1-q^{2 n}}, \quad \Sigma \frac{(2 n+1) a_{n} q^{2 n+1}}{1-q^{4 n+2}}, \ldots
$$

Fin inger from

$$
-\log k^{\prime}=8 \sum_{0}^{\infty} \frac{q^{2 n+1}}{(2 n+1)\left(1-q^{4 n+2}\right)}{ }^{\dagger}
$$

we deduce

$$
-\log _{q=1} k^{\prime} \sim \frac{\pi^{2}}{2(1-q)}
$$

and from

$$
\begin{aligned}
& \frac{2 \omega}{\pi} \sqrt{ }\left(\wp_{1}-e_{3}\right)=\operatorname{cosec} \frac{u \pi}{2 \omega}+4 \Sigma \frac{q^{2 n+1}}{1-q^{2 n+1}} \sin \left\{(2 n+1) \frac{u \pi}{2 \omega}\right\} \ddagger \\
& \frac{2 \omega}{\pi} \sqrt{ }\left(\wp_{u}-e_{3}\right) \sim \frac{4}{1-q} \Sigma \frac{\sin \left\{(2 n+1) \frac{u \pi}{2 \omega}\right\}}{2 n+1}= \pm \frac{\pi}{1-q},
\end{aligned}
$$

according to the value of $u$. In the last equation we must suppose that $\omega$ is constant and that $\omega^{\prime}$ varies in such a way that $q$ tends to $l$ along the real axis.

In an interesting note recently published in the Messenger of Mathematics, $\$$ Prof. Bromwich establishes the asymptotic equality

$$
f(\theta)=\sum_{1}^{\infty} \frac{(-)^{n-1}}{\sinh n \theta} \sim \frac{\log 2}{\theta}
$$

for $\theta=0$. This result follows immediately from what precedes if we write $q$ for $\theta$. I shall refer later on to Prof. Bromwich's further results.

4. I shall now consider some examples of the use of Theorem I. $a 1$.

(i.) Suppose that $f_{n}(x)=x^{n}$, and that the region of variation of $x$ is a triangle formed by joining 0 and 1 to any point inside the unit circle.

It is easily verified that a constant $K$ (depending only on the triangle) can be found such that for all points within or on the boundary of the triangle

$$
\frac{|1-x|}{1-|x|}<K \text {. }
$$

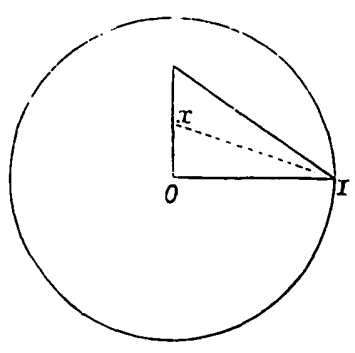

Hence, if $|x|=r$,

$$
\sum_{m}^{n}\left|f_{\nu}(x)-f_{\nu+1}(x)\right|=\sum_{m}^{n} r^{\nu}|1-x|<K \sum_{m}^{n} r^{\nu}(1-r)<K
$$

- Matĭ. Annalen, Bd. xur.

$\dagger$ Fundamenta Nova, l.c.

‡ Ealphen, Fonctions Elliptiques, t. I., p. 431.

\$ "Some Contributions to the Theory of 'Two Electrified Spheres," Messenger, Vol. xxxv., p. 1. 
and the conditions of the theorem are satisfied. We thus obtain Pringsheim's generalisation of Abel's theorem.*

(ii.) The theorem may be applied to $q$-series such as those previously considered when $q$ moves (let us say) alony a radiue vector to a rational point on the unit circle, i.e., a point eriba". where $a$ and $b$ are integers. Take, e.g., the series for $\log k$ considered above, $\dagger$ and suppose that $\eta=r e^{\pi i b, u}$, where $b$ is even and $a$ odd, and that $r$ tends to unity along the radius vector $(0,1)$. Then none of the terms of the series become infinite in the limit; also

$$
\sum_{1}^{\infty} \frac{(-q)^{n}}{n\left(1+q^{n}\right)}=\sum_{s=1}^{n} \sum_{m=0}^{\infty} \frac{(-q)^{m a+s}}{(m a+s)\left(1+q^{m a+s}\right)}=\sum_{s=1}^{n}(-)^{s} q^{s} e^{s+i b i a} F_{s}\left(r^{m}\right),
$$

where

$$
F_{s}(\rho)=\sum_{0}^{\infty} \frac{(-\rho)^{m}}{(m a+s)\left(1+\rho^{m+s i n} e^{s-i b / a}\right)} \text {. }
$$

This lust series satisfies the criteria of $I$. a 1 for uniform convergence throughout the interval $(0,1)$ of values of $\rho$. For, if $a_{m}=(-)^{m} /(m a+s), \Sigma a_{m}$ is convergent. Also, if

$$
\begin{gathered}
f_{m}(\rho)=\frac{\rho^{m}}{1+\rho^{m+s / a} e^{s+i b / a},} \\
f_{m}(\rho)-f_{m+1}(\rho)=\frac{\rho^{m}(1-\rho)}{\left(1+A \rho^{m+s a}\right)\left(1+A \rho^{m+1+s / a}\right)^{\prime}}
\end{gathered}
$$

where $A=e^{a r b l a}$. Now

$$
\left|1+A \rho^{m+o a}\right|=\sqrt{ }\left\{1+\rho^{2(m+s / a)}+2 \rho^{m+s, a} \cos (s \pi b / a)\right\} \text {. }
$$

If $\cos (s \pi b / a)>0$, this is greater than unity: if $\cos (s \pi b / x)<0$, it has a minimum when $\rho^{m+s / a}=-\cos (s \pi b / a)$, this minimum being $|\sin (s \pi b / a)|$. And in any case

$$
\left|f_{m}(\rho)-f_{m+1}(\rho)\right|<h_{f}^{\prime \prime}(1-\rho) \text {, }
$$

from which it follows at once that the conditions of I. $a 1$ are sutisfied.

Hence the original series for $\log k$ converges uniformly when $q=v e^{\pi i b / a}, 0 \leqslant r \leqslant 1$. For $r=1$ it assumes the form

$$
2 \log 2+\frac{\pi i b}{2 a}+2 \sum_{1}^{\infty} \frac{(-)^{n}}{n}\left(1+i \tan \frac{n \pi b}{a}\right)=\frac{\pi i b}{a}+2 i \sum_{1}^{\infty}-\frac{1}{n} \tan \frac{n \pi b}{a},
$$

and this is therefore the value to which $\log k$ tends as $r$ approaches unity. The series on the right may be summed in finite terms. $\neq$

5. In a passage in his Vorlesungen iiber Integrale, which has doubtless puzzled many readers besides myself, Kronecker apparently essays to prove a theorem designed to be a generalisation of Abel's theorem somewhat on the lines of Theorem I. a, except that there is no mention of uniform convergence. The whole passage is obscure; but the suggested

- Müuchener Sitzungsberichte, l.c.

$+\$ 3$, iii.

† See H. J. S. Smith, " On some Discontinuous Series considered by Riemunn" (Messenger, Vol. xI., pp. 1-11 ; Collected Math. Papers, Vol. Ir., p. 312) ; Dedekind's Note in Riemann's Werlie, pp. $42 \bar{i}-447$; G. H. Hardy, "Note on the Limiting Values of the Elliptic Modular Functions," Quturterly Jourmal, Vol. xxxiv., pp. 76-86. 
theorem seems to be as follows:-—" "If

(i.) $\Sigma a_{n}$ is a convergent series,

(ii.) the functions $f_{n}(x)$ are positive and continuous throughout $(a, A)$,

(iii.) $f_{n}(x) \geqslant f_{n+1}(x)$,

(iv.) $\lim _{x=A} f_{m}(x)=\lim _{x=A} f_{n}(x)$, for all values of $m$ and $n$,

then $\Sigma a_{n} f_{n}(x)$ will be convergent and continuous for $x=A . "$

My criticisms on the passage are in brief (i.) that the conditions are redundant, the fourth of them being quite unnecessary and having nothing to do with the essence of the matter; and (ii.) that the proof is altogether unsound. The unsoundness of the proof appears to have arisen from a mistaken idea of the importance of condition (iv.). Kronecker argues as follows. Starting from Abel's partial summation lemma, the origin of all these theorems, viz.,

$$
c_{0} f_{0}+\sum_{1}^{n}\left(c_{\nu}-c_{\nu-1}\right) f_{v-1}=\sum_{1}^{n} c_{\nu}\left(f_{v-1}-f_{\nu}\right)+c_{n} f_{n},
$$

and putting

$$
c_{\nu}=-\left(a_{\nu}+a_{\nu+1}+\ldots\right) \text {, }
$$

he deduces

$$
\begin{aligned}
-f_{0} \sum_{0}^{\infty} a_{\nu}+\sum_{1}^{n} a_{\nu-1} f_{v-1} & =-\sum_{1}^{n}\left(f_{v-1}-f_{v}\right) \sum_{v}^{\infty} a_{\kappa}-f_{n} \sum_{n}^{\infty} a_{\kappa} \\
& =-\left(f_{0}-f_{n}\right) M I_{n}-f_{n} \sum_{n}^{\infty} a_{k},
\end{aligned}
$$

where $M_{n}$ lies between the least and greatest of the values of

$$
\sum_{\nu}^{\infty} a_{x} \quad(\nu=1,2, \ldots, n) \text {. }
$$

Making $n$ tend to infinity, and observing that $\sum_{1}^{\infty} a_{\nu-1} f_{\nu-1}$ is convergent, we obtain

$$
-f_{0} \sum_{n}^{\infty} a_{\nu}+\sum_{1}^{\infty} a_{\nu-1} f_{\nu-1}=-\left(f_{0}-\lim _{n=0} f_{n}\right) M,
$$

where $M$ lies between the least and greatest of all the values of $\sum_{\nu}^{\infty} a_{x}$.

He then makes $x$ tend to $A$, and (unless his meaning has been entirely obscured by misprints), arguès that, because

$$
\lim _{x=A} f_{0}=\lim _{x=A} f_{n}
$$

- I have altered Kronecker's notation 80 as to agree with my own (Kronecker, l.c., pp. 88, 89). 
for all values of $n$, therefore

$$
\lim _{x=A}\left(f_{0}-\lim _{n=\infty} f_{n}\right)=0 ;
$$

and therefore $\quad \lim _{x=A} \sum_{1}^{\infty} a_{\nu-1} f_{\nu-1}=\lim _{x=A} f_{0} \times \sum_{0}^{\infty} a_{\nu}$.

But it is obvious that all that he is justified in asserting is that

$$
\lim _{x=A} f_{0}=\lim _{n=\infty}\left(\lim _{x=A} f_{n}\right)
$$

and not

$$
\lim _{x=A} f_{0}=\lim _{x=A}\left(\lim _{n=\infty} f_{n}\right) \text {, }
$$

the two repeated limits only being equal in exceptional circumstances. And, in fact, in the very simplest case, when $f_{n}(x)=x^{n}$ and $A=1$,

$$
\lim _{n=\infty} \lim _{x=1} x^{n}=1, \quad \lim _{x=1} \lim _{n=\infty} x^{n}=0 ;
$$

so that his argument does not even suffice to prove Abel's theorem itself. And a careful examination of the passage will, I think, lead any reader to the conclusion that the flaw in it is fundamental and not to be repaired by any alterations merely of detail.

6. I shall now consider the case in which the series $\Sigma a_{n}$ is divergent but summable by Cesàro's method of mean values. I use the following notation and terminology. We shall say that $\Sigma a_{n}$ is summable if

$$
\frac{s_{0}+s_{1}+\ldots+s_{n}}{n+1}
$$

where

$$
s_{n}=a_{0}+a_{1}+\ldots+a_{n},
$$

tends to a finite limit for $n=\infty$; and, if the terms $a_{n}$ are functions of a variable $x$, and the convergence of this mean value to its limit is uniform throughout a certain interval or region, we shall say that $\Sigma a_{n}$ is uniformly summable. It is evident that the sum of a uniformly summable series of continuous terms is a continuous function of $x$.

Theorem 2.-If the functions $f_{n}$ are finite, real, and positive, and $f_{n}-f_{n+1}$ and $f_{n}-2 f_{n+1}+f_{n+2}$, their first and second differences, are positive for $0 \leqslant x \leqslant 1$ and for all values of $n$, and if the series $\Sigma a_{n}$ is summable, then the series $\Sigma a_{n} f_{n}$ is uniformly summable throughout $(0,1)$.

Coroldary.-If the functions $f_{n}$ are continuous, the sum of the series $\Sigma a_{n} f_{n}$ is a continuous function of $x$.

The proof of this theorem presents somewhat greater difficulties than those of the simpler theorems of $\S 2$. We shall find it a necessary preliminary to establish a series of lemmas. 
Lemma 1.-If $s_{n}$ tends uniformly to a limit $s$, the series $\Sigma a_{n}$ is uniformly summable and has the sum s.

If we omit " uniformly," this is a well known theorem* asserting the consistency of the new definition with the old. The insertion of "uniformly" in no way affects the proof.

$$
\text { Lemma 2.-If } \quad \lim \frac{s_{0}+s_{1}+\ldots+s_{n}}{n+1}=0,
$$

we can determine a series of positive quantities $\epsilon_{1}, \epsilon_{2}, \ldots$, whose limit is zero, such that

for all values of $r$.

$$
\left|\frac{s_{p}+s_{p+1}+\ldots+s_{p+r}}{p+r+1}\right|<\epsilon_{p}
$$

For we may write $s_{0}+s_{1}+\ldots+s_{n}=(n+1) \eta_{n}$, where $\lim \eta_{n}=0$. And then

$$
s_{p}+s_{p+1}+\ldots+s_{p+r}=(p+r+1) \eta_{p+r}-p \eta_{p-1},
$$

from which the lemma follows; for we can choose $p$ so that, for $\nu \geqslant p-1$, $\left|\eta_{\nu}\right|<\epsilon$, however small be $\epsilon$, and then

$$
\left|\frac{s_{p}+s_{p+1}+\ldots+s_{p+r}}{p+r+1}\right|<2 \epsilon
$$

for all values of $r$. In particular, as is well known,

$$
\lim s_{p} /(p+1)=0 .
$$

Lemma 3.-If $f_{n}$ is finite, real, and positive and $f_{r} \geqslant f_{n+1}$ for all values of $n$ and $x$, and

then

$$
\lim \frac{s_{0}+s_{1}+\ldots+s_{n}}{n+1}=0 ;
$$

$$
\lim \frac{s_{0} f_{0}+s_{1} f_{1}+\ldots+s_{n} f_{n}}{n+1}=0
$$

uniformly for all values of $x$.

For

$$
\begin{aligned}
s_{0} f_{0}+\ldots+s_{n} f_{n} & =\sum_{\nu=0}^{n-1}\left(s_{0}+\ldots+s_{\nu}\right)\left(f_{\nu}-f_{\nu+1}\right)+\left(s_{0}+\ldots+s_{n}\right) f_{n} \\
& =\left(\sum_{\nu=0}^{r-1}+\sum_{r}^{n-1}\right)\left(s_{0}+\ldots+s_{\nu}\right)\left(f_{\nu}-f_{\nu+1}\right)+\left(s_{0}+\ldots+s_{n}\right) f_{n} \\
& =\left(f_{0}-f_{r}\right) M_{0, r-1}+f_{r} M_{r, n},
\end{aligned}
$$

* See, e.g., Bromwich and Hardy, Proceedings, Vol. II., p. 172.

sEr. 2. vor. 4 . No. 928. 
where $M_{0, r-1}$ lies between the least and greatest of

$$
s_{0}, s_{0}+s_{1}, \ldots, s_{0}+s_{1}+\ldots+s_{r-1},
$$

and $M_{r, n}$ between the least and greatest of

$$
s_{0}+s_{1}+\ldots+s_{r}, \ldots, s_{0}+s_{1}+\ldots+s_{n} .
$$

Let $\epsilon$ be an assigned positive small quantity. We can choose $r$ so that for $\nu \geqslant r$

$$
\left|\frac{s_{0}+s_{1}+\ldots+s_{v}}{\nu+1}\right|<\epsilon,
$$

and, a fortiori, $\quad\left|\frac{s_{0}+s_{1}+\ldots+s_{v}}{n+1}\right|<\epsilon$

for $n \geqslant \nu \geqslant r$; and therefore we can choose $r$ so that

$$
\left|\frac{M_{r, n}}{n+1}\right|<\epsilon
$$

for all values of $n \geqslant r$. But when $r$ is fixed we can obviously choose $n$ so that

$$
\left|\frac{M_{0, r-1}}{n+1}\right|<\epsilon .
$$

When $r$ and $n$ are thus chosen

$$
\left|\frac{s_{0} f_{0}+s_{1} f_{1}+\ldots+s_{n} f_{n}}{n+1}\right|<2 M \epsilon,
$$

where $M$ is the maximum of $f_{0}(x)$. The lemma is therefore proved.

Lemma 4.-If the conditions of 3 are satisfied except that

$$
\lim _{n=\infty} \frac{s_{0}+s_{1}+\ldots+s_{n}}{n+1}=s(\neq 0) \text {, }
$$

then

$$
\lim _{n=\infty} \frac{s_{0} f_{0}+s_{1} f_{1}+\ldots+s_{n} f_{n}}{n+1}=s \lim _{n=\infty} f_{n} ;
$$

but the convergence to this limit will in general not be uniform.

For let $s_{0}=s+t_{0}, s_{1}=s+t_{1}, \ldots$ Then

$$
\lim _{n=\infty} \frac{t_{0}+t_{1}+\ldots+t_{n}}{n+1}=0 ;
$$

and therefore

$$
\frac{t_{0} f_{0}+t_{1} f_{1}+\ldots+t_{n} f_{n}}{n+1}
$$


1906.] Theorems connected with Abel's theoren on POWER series. 259 converges uniformly to zero. Also

$$
\lim _{n=\infty} \frac{s\left(f_{0}+f_{1}+\ldots+f_{n}\right)}{n+1}=s \lim _{n=\infty} f_{n} ;
$$

but the convergence to this limit will not in general be uniform unless $f_{n}$ converges to its limit uniformly, which will not generally be the case.

$$
\text { Lemma 5.-If } \quad \lim _{n=\infty} \frac{s_{0}+s_{1}+\ldots+s_{n}}{n+1}=0,
$$

and the $f_{n}$ 's satisfy the further condition

$$
f_{n}-f_{n+1} \geqslant f_{n+1}-f_{n+2}
$$

for all values of $n$ and $x$ in question, then the series

is uniformly convergent.

$$
\sum_{0}^{\infty} s_{n}\left(f_{n}-f_{n+1}\right)
$$

In the first place

$$
f_{0}-f_{n}=\left(f_{0}-f_{1}\right)+\ldots+\left(f_{n-1}-f_{n}\right) \geqslant n\left(f_{n-1}-f_{n}\right) .
$$

Hence a constant $K$ can be assigned so that for all values of $x$ and $n$

$$
f_{n-1}-f_{n}<K / n \text {. }
$$

$$
\text { Now } \begin{aligned}
s_{p}\left(f_{p}-f_{p+1}\right)+s_{p+1}\left(f_{p+1}-f_{p+2}\right) & +\ldots+s_{q-1}\left(f_{q-1}-f_{q}\right) \\
=s_{p}\left(f_{p}-2 f_{p+1}+f_{p+2}\right) & +\left(s_{p}+s_{p+1}\right)\left(f_{p+1}-2 f_{p+2}+f_{p+9}\right) \\
& +\ldots \ldots l l l \\
& +\left(s_{p}+s_{p+1}+\ldots+s_{q-2}\right)\left(f_{q-2}-2 f_{q-1}+f_{q}\right) \\
& +\left(s_{p}+s_{p+1}+\ldots+s_{q-1}\right)\left(f_{q-1}-f_{q}\right)
\end{aligned}
$$

the modulus of which is less than

$$
\begin{gathered}
\epsilon_{p}\left\{(p+1)\left(f_{p}-2 f_{p+1}+f_{p+2}\right)+(p+2)\left(f_{p+1}-2 f_{p+2}+f_{p+3}\right)+\ldots\right. \\
\left.\cdots+(q-1)\left(f_{q-2}-2 f_{q-1}+f_{q}\right)+q\left(f_{q-1}-f_{q}\right)\right\} \\
=\epsilon_{p}\left\{p\left(f_{p}-f_{p+1}\right)+f_{p}-f_{q}\right\}<\epsilon_{p}\{K+2 M\},
\end{gathered}
$$

where $M$ is the maximum of $f_{0}(x)$. The lemma is therefore proved. 
Lemas 6.-If the $f_{n}$ 's satisfy the conditions of 5 , but

$$
\lim _{n=\infty} \frac{s_{0}+s_{1}+\ldots+s_{n}}{n+1}=s(\neq 0),
$$

the series

$$
\sum_{0}^{\infty} s_{n}\left(f_{n}-f_{n+1}\right)
$$

is convergent (but, in general, not uniformly convergent).

$$
\text { Let } \quad s_{n}=s+t_{n} ;
$$

then, by 3 , the series $\Sigma t_{n}\left(f_{n}-f_{n+1}\right)$ is uniformly convergent. On the other hand, the series $\Sigma s\left(f_{n}-f_{n+1}\right)$ is convergent, but not uniformly convergent, unless $f_{n}$ tends to its limit uniformly.

7. Proof of Theorem 2.-Let $s$ be the sum of the divergent series $\Sigma a_{n}$, and let

$$
a_{0}^{\prime}=a_{0}-s, a_{1}^{\prime}=a_{1}, a_{2}^{\prime}=a_{2}, \ldots, s_{n}^{\prime}=a_{0}^{\prime}+a_{1}^{\prime}+\ldots+a_{n}^{\prime}=s_{n}-s ;
$$

then $\Sigma a_{n}^{\prime}$ is summable, and its sum is zero; i.e.,

$$
\lim \frac{s_{0}^{\prime}+s_{1}^{\prime}+\ldots+s_{n}^{\prime}}{n+1}=0 .
$$

By Lemma 3,

$$
\frac{s_{0}^{\prime} f_{0}+s_{1}^{\prime} f_{1}+\ldots+s_{n 2}^{\prime} f_{n}}{n+1}
$$

tends uniformly to 0 for $n=\infty$; and, by Lemma 5 , the series

$$
\Sigma s_{n}^{\prime}\left(f_{n}-f_{n+1}\right)
$$

is uniformly convergent. Hence, if

$$
S_{n}^{\prime}=\sum_{0}^{n} s_{\nu}\left(f_{\nu}-f_{\nu+1}\right)
$$

$S_{n}^{\prime}$ tends uniformly to a limit for $n=\infty$, and so, by Lemma 1 ,

does the same.

$$
\frac{S_{n}^{\prime}+S_{1}^{\prime}+\ldots+S_{n}^{\prime}}{n+1}
$$

Now $\quad a_{\nu}^{\prime} f_{\nu}=\left(s_{\nu}-s_{\nu-1}^{\prime}\right) f_{\nu}=s_{\nu}^{\prime} f_{\nu}-s_{\nu-1}^{\prime} f_{\nu-1}+s_{\nu-1}^{\prime}\left(f_{\nu-1}-f_{\nu}\right)$.

Hence, if $\sigma_{n}=a_{0} f_{0}+a_{1} f_{1}+\ldots+a_{n} f_{n}, \sigma_{n}^{\prime}=a_{0}^{\prime} f_{0}+a_{1}^{\prime} f_{1}+\ldots+a_{n}^{\prime} f_{n}$,

$$
\sigma_{n}^{\prime}=s_{n}^{\prime} f_{n}+\sum_{1}^{n} s_{v-1}^{\prime}\left(f_{v-1}-f_{v}\right)=s_{n}^{\prime} f_{n}+S_{n-1}^{\prime} \text {, }
$$

and $\frac{\sigma_{0}^{\prime}+\sigma_{1}^{\prime}+\ldots+\sigma_{n}^{\prime}}{n+1}=\frac{s_{0}^{\prime} f_{0}+\ldots+s_{n} f_{n}}{n+1}+\left(\frac{n}{n+1}\right) \frac{S_{0}^{\prime}+S_{1}^{\prime}+\ldots+S_{n-1}}{n}$, 
1906.] Theorems connected with Abel's theorem on power series. 261

and therefore tends uniformly to a limit for $n=\infty$. But

$$
\frac{\sigma_{0}+\sigma_{1}+\ldots+\sigma_{n}}{n+1}=s f_{0}+\frac{\sigma_{0}^{\prime}+\sigma_{1}^{\prime}+\ldots+\sigma_{n}^{\prime}}{n+1}
$$

and therefore also tends uniformly to a limit for $n=\infty$. Hence the series $\Sigma a_{n} f_{n}$ is uniformly summable, and, if the functions $f_{n}$ are continuous, its sum is a continuous function of $n$. The theorem is therefore proved.

8. In order to show more precisely the relations of the preceding lemmas and theorem I take a very simple example.

Let

$$
a_{0}=1, \quad a_{1}=-2, \quad a_{2}=2, \quad a_{3}=-2, \quad \ldots,
$$

so that

$$
s_{2,4}=1, \quad s_{2 n+1}=-1 \text {, }
$$

and

and suppose $f_{n}(x)=x^{*}$. Then

$$
\lim \frac{s_{0}+s_{1}+\ldots+s_{n}}{n+1}=0 \text {; }
$$

$$
\begin{gathered}
s_{n} f_{n}=(-)^{n} x^{n}, \\
s_{0} f_{0}+s_{1} f_{1}+\ldots+s_{n} f_{n}=\frac{1+(-)^{n} \cdot x^{n+1}}{(n+1)(1+x)},
\end{gathered}
$$

which converges uniformly to 0 for $n=\infty$ (Lemma 3 ).

(ii.) Again $\sum_{1}^{n} s_{\nu-1}\left(f_{\nu-1}-f_{\nu}\right)=\sum_{1}^{n}(-)^{\nu-1} x^{\nu-1}(1-x)=(1-x)\left\{1+(-)^{n-1} x^{n}\right\} /(1+x)$,

which tends vinformly to $(1-x) /(1+x)$ for $n=\infty$ (Lemma 5). For, although $x^{n}$ does not tend uniformly to its limit,

$$
x^{n}-x^{n+1}-\left(x^{n+1}-x^{n+2}\right)=x^{n}(1-x)^{2} \geqslant 0,
$$

and

$$
1-x^{n+1}=(1-x)+\left(x-x^{2}\right)+\ldots+\left(x^{n}-x^{n+1}\right) \geqslant(n+1)\left(x^{n}-x^{n+1}\right) \text {, }
$$

so that

$$
x^{n}(1-x)<\frac{1}{n+1}
$$

and therefore does tend uniformly to zero.

(iii.) Finally, $\sigma_{n}=1-2 x+2 x^{2}-\ldots+(-)^{n} 2 x^{n}=\frac{1-x}{1+x}+2(-)^{n} \frac{x^{n+1}}{1+x}$,

and

$$
\sigma_{0}+\sigma_{1}+\ldots+\sigma_{n}=\frac{1-x}{1+x}+\frac{2\left\{x+(-)^{n} x^{n+2}\right\}}{(n+1)(1+x)^{2}}
$$

which tends uniformly to $(1-x) /(1+x)$ for $n=\propto$ (Theoresn 2$)$.

If the conditions were altered by changing $a_{0}$ into $1+\alpha(\alpha \neq 0)$, we should have

and

$$
s_{n} f_{n}=\left\{a+(-1)^{n}\right\} x^{n}
$$

where

$$
\begin{aligned}
& \frac{s_{0} f_{0}+s_{1} f_{1}+\ldots+s_{n} f_{n}}{n+1}=\phi+\begin{array}{l}
1+(-)^{n} x^{n+1} \\
(n+1)(1+x)
\end{array} \\
& \phi=\frac{\alpha}{n+1} 1-x^{n+1} \quad(x<1), \\
& \phi=a \quad(x=1),
\end{aligned}
$$

and the convergence of $\phi$ to its limit is not uniform (Lemma 4). Similarly $\Sigma s_{\nu-1}\left(f_{\nu-1}-f_{\nu}\right)$ is increased by the addition of the non-uniformly convergent series $\Sigma \alpha\left(x^{n-1}-x^{n}\right)$ (Lemma 6$)$; but it is easily verified that the uniformity of convergence which is prescribed by Theorem 2 is not affected, the two non-uniformities (so to say) cancelling oue another. 
9. Applications of Theorem 2.-(i.) If $f_{n}(x)=x^{n}$,

$$
f_{n}-2 f_{n+1}+f_{n+2}=x^{n}(1-x)^{2} \geqslant 0
$$

for $0 \leqslant x \leqslant 1$ and all values of $n$. Hence, if $\Sigma a_{n}$ is summable, $\Sigma a_{n} x^{n}$ is uniformly summable for $0 \leqslant x \leqslant 1$; and its sum is a continuous function of $x$ for $x=1$, which is Frobenius's theorem cited in $\$ 1$.

(ii.) If $f_{n}(x)=n^{-x}(n \geqslant 1, x \geqslant 0)$, it is easy to see that the first and second differences of $f_{11}$ are positive (or zero). Hence we obtain the theorem that, if $\sum_{1}^{\infty} a_{n}$ is summable, $\sum_{1}^{\infty} a_{n} n^{-x}$ is uniformly summable for all positive values of $a$, inclnding zero, and its sum is a continuous function of $x$ for $x=0$. That is to say

$$
\lim _{x=0}\left(\frac{a_{1}}{1^{x}}+\frac{a_{2}}{2^{x}}+\frac{a_{3}}{3^{x}}+\ldots\right)=\lim _{n=\infty} \frac{s_{1}+s_{1}+\ldots+s_{n}}{n+1}
$$

if the latter limit exists. For example,

$$
\lim _{x=0}\left(i_{1}^{x}-\frac{1}{2^{x}}+\frac{1}{3^{x}}-\ldots\right)=\frac{1}{2} .
$$

(iii.) If

$$
\begin{aligned}
& f_{n}(q)=\frac{q^{2}}{1+q^{n}} \quad(0 \leqslant q \leqslant 1), \\
& f_{n}-2 f_{n+1}+f_{n+2}=\frac{q^{n}(1-q)^{2}\left(1-q^{n+1}\right)}{\left(1+q^{n}\right)\left(1+q^{n+1}\right)\left(1+q^{n+2}\right)} \geqslant 0 \text {. }
\end{aligned}
$$

Hence, if $\Sigma a_{n}$ is summable, $\Sigma a_{n} q^{n} /\left(1+q^{n}\right)$ is uniformly summable for $0 \leqslant q \leqslant 1$, and represente a continuous function of $q$, in particular for $\eta=1$.

For instance, from the formula

$$
\frac{2 k^{\prime} K}{\pi}=1-\frac{4 q}{1+q}+\frac{4 q^{3}}{1+q^{3}}-\frac{4 q^{5}}{1+q^{5}}+\ldots
$$

we deduce that

$$
\lim _{q=1} \frac{2 k^{\prime} T}{\pi}=1-4\left(\frac{1}{2}-\frac{1}{2}+\ldots\right)=1-4 \cdot \frac{1}{4}=0 . \dagger
$$

(iv.) Consider the series

$$
\frac{q}{1-q^{2}}-\frac{2 q^{2}}{1-q^{4}}+\frac{3 q^{3}}{1-q^{6}}-\ldots
$$

whose sum is easily found + to be

$$
\frac{\hbar}{2 \pi^{2}}\left(E-k^{\prime 2} K\right) \text {. }
$$

We may write this in the form

$$
\frac{q}{1-q^{2}} \Sigma a_{n} f_{n}(q)
$$

where

$$
a_{n}=(-1)^{n} \quad \text { and } \quad f_{n}(q)=\frac{(n+1) q^{n}}{1+q^{2}+\ldots+q^{2 n}} \text {, }
$$

and it is easy to verify that the first and second differences of $f_{n}$ are positive. Hence $\sum a_{n} f_{n}$ is uniformly summable. For $q=1$ it takes the form

$$
1-1+1-\ldots=\frac{1}{2} \text {. }
$$

- Fundamenta Nova, $\$ 40,(6)$.

+ Strictly speaking, the divergent series should be written

$$
\frac{1}{2}+0-\frac{1}{2}-0+\frac{1}{2}+0-\frac{1}{2}-\ldots .
$$

$\ddagger$ E.y., by muking $r=\frac{1}{2} \pi$ in formula (1) of $\$+1$ of the Fundamenta Nova. 
We deduce that

$$
K\left(E-k^{\prime 2} K\right) \sim \frac{\pi^{2}}{2(1-q)}
$$

for $q=1$.

10. It would be easy to multiply instances of interesting applications of Theorem 2. Those which I have given are fair examples of some of the simplest types which naturally occur, and the length of this paper forbids that I should attempt to treat them in a more systematic manner. I shall conclude by indicating briefly certain actual or possible further generalisations.

In the first place we may at once enunciate

Theonem $2 a 1$. - The conclusions of Theorem 2 (and the lemmas proliminary to it) are still valid if the functions $f_{n}(x)$ are not restricted to be real and positive, and the condition that the first and second differences of the functions are not negative is replaced by the conditions

$$
\sum_{m}^{n}\left|f_{\nu}-f_{\nu+1}\right|<K, \quad \sum_{m}^{n}(\nu+1)\left|f_{\nu}-2 f_{\nu+1}+f_{\nu+2}\right|<K,
$$

for all values of $m, n$, and $x$.

The course of the proof is unaffected save for slight modifications in the case of Lemmas 3 and 5.

Consider, for example, the series

$$
\vartheta_{4}(v, q)=1+2 \sum_{1}^{\infty}(-)^{n} q^{\prime \prime} \cos 2 n \pi v .
$$

Taking $a_{n}=2(-)^{n} \cos 2 n \pi v(n>0)$ and $f_{n}=q^{n^{2}}$, we may verify without difficulty that the conditions of the theorem are satisfied. Since the series

$$
1-2 \cos 2 \pi v+2 \cos 4 \pi v-\ldots
$$

has the sum zero when summed by Cesàro's method, we deduce that

$$
\lim _{q=1} \hat{S}_{4}(v, q)=0 . *
$$

Theorem 2 a 2.-The preceding conclusions are not affected if the terms of the series $\Sigma a_{n}$ are functions of $x$, provided the series be uniformly summable.

A much more interesting and more difficult question is that of the extension of Theorem II. to cases in which the summation of $\Sigma a_{n}$ requires

- Sce Borel, Leçons sur les Séries divergmutes, p. 7 ; L. Fejér, Math. Amnalen, Bd. Lvir., p. 66 ; Hardy, "Note on Divergent Fourier Series," Messenger, Vol. xxxmr., p. 144 . I refer later to Herr Fejér's investigations. 
one of the extended forms of the mean value process, e.g., when, if

$$
s_{n}^{(1)}=\frac{s_{0}+s_{1}+\ldots+s_{n}}{n+1},
$$

$s_{n}^{(1)}$ oscillates for $n=\infty$, but

has a limit.

$$
s_{n}^{(2)}=\frac{s_{0}^{(1)}+s_{1}^{(1)}+\ldots+s_{n}^{(1)}}{n+1}
$$

The following more general theorem is naturally suggested, and I have no doubt that it is true. We define "summable" to mean "summable by $k$ repetitions of the mean value process." Then,

If the first, second, ..., $(k+1)$-th differences of the functions $f_{n}(x)$ are positive (or zero) for all values of $x$ and $n$ in question, and the series $\Sigma a_{n}$ is summable, then the series $\Sigma a_{n} f_{n}(x)$ is uniformly summable, and therefore its sum is a continuous function of $x$

-with corollaries and generalisations in every way analogous to those of Theorems I. $a$ and II. Such a theorem would be related to Hölder's extensions of Frobenius's theorem as is II. to Frobenius's and I. $a$ to Abel's theorem. But I have not up to the present succeeded in overcoming the algebraical difficulties attendant upon a complete and rigorous proof.

In the most interesting cases Theorem II. is generally sufficient. But the latter theorem does not cover such cases as those in which $\Sigma a_{n}$ is a series like $1-2+3-4+\ldots$ or $1^{2}-2^{2}+3^{2}-4^{2}+\ldots$.

An example in which a result more general than that of II. is needed may be found in the theory of two electrified spheres. In the paper already referred to, Prof. Bromwich, seeking a rigorous proof of Lord Kelvin's theorem that the force acting between two spheres in contact and at potential $V$ is $\frac{3}{6} V^{2}(\log 2-4)$, requires to show that, for small values of $\theta$,

$$
f(\theta)=\Sigma \frac{(-)^{n-1}}{\sinh n \theta}=\frac{\log 2}{\theta}-\frac{1}{2} \frac{1}{4} \theta+\ldots
$$

The first approximation was established in $\$ 3$ (iv.). To obtain the second we must prove that

$$
\lim _{\theta 00} \frac{1}{\theta} \Sigma(-)^{n-1}\left(\frac{1}{n \theta}-\frac{1}{\sinh \mu \theta}\right)=\frac{1}{2} \text {. }
$$

The limiting form of the series is $\frac{1}{6}(1-2+3-4+\ldots)$,

which is summable by tuo repetitions of the mean value process, and has the sum $\frac{1}{2} \frac{1}{6}$. Here we could take $a_{n}=(-)^{n-1} n$ and $f_{n}(\theta)=\frac{1}{n \theta}\left(\frac{1}{n \theta}-\frac{1}{\sinh n \theta}\right)$, and so obtain the result desired.

Although I have not succeeded in proving the suggested general theorem, I have, starting from a theorem of Herr Fejér's, succeeded in proving a number of theorems of a more special character which do enable us to deal effectively with cases such as these: e.g., to assign the limit of

$$
q+\frac{2 q^{2}}{1+q^{2}}+\frac{3 q^{3}}{1+q^{3}}-\ldots
$$


1906.] Theorems connected with Abel's theorem on POWer series. 265

for $q=1$. I confine myself at present to stating one of these theorems. Herr Fejér's theorem (modified so as to sorrespond to Theorem $2^{*}$ ) runs as follows :- If

(i.) $\Sigma a_{n}$ is suminable (to the sum $s$ ),

(ii.) the functions $f_{n}(x)$ and their first and second differences are positive (or zero),

(iii.) $\Sigma n f_{n}(x)$ is convergent for $x>0$,

(iv.) $\lim _{x=0} f_{n}(x)=1$ for all values of $n$,

then $\Sigma a_{n} f_{n}(x)$ is absolutely convergent for $x>0$, and its limit for $x=0$ is $s$.

The more general theorem is that the same conclusion holds when $k$ repetitions of the mean value process are necessary in order to sum the series $\Sigma n_{n}$, and

(ii.)' the first, second, ..., $(k+1)$-th differences of the functions $f_{n}(x)$ are positivc (or zero),

(iii.)' $\Sigma u^{k} f_{n i}^{\prime}(x)$ is absolutely convergent.

The proof is not difficult. The other theorems relate to cases in which condition (ii.) or (ii.)' is not satisfied. I have included proofs of these theorems in a paper which will be published in the Muthematische Annalen.

- The conditions actually stated by Herr Fejér differ from the above in the restriction of $f_{n}(x)$ to be of the form $\phi(n x)$, and the substitution for (ii.) and (iii.) of the conditions

$$
|\phi(t)|<\frac{K}{t^{2}+\rho}, \quad\left|\phi^{\prime \prime}(t)\right|<\frac{K}{t^{2}+\rho},
$$

where $\rho>0$. The proof of the theorem as I state it may be made a good deal simpler than Herr Fejér's proof. 